\title{
A Comparative Analysis of Augmented Reality Frameworks Aimed at the Development of Educational Applications
}

\author{
Fabrício Herpich ${ }^{1}$, Renan Luigi Martins Guarese², Liane Margarida Rockenbach Tarouco ${ }^{1}$ \\ ${ }^{1}$ Graduate Program in Informatics in Education, Federal University of Rio Grande do Sul (UFRGS), Porto Alegre, Brazil \\ ${ }^{2}$ Institute of Informatics, Federal University of Rio Grande do Sul (UFRGS), Porto Alegre, Brazil \\ Email: fabricio_herpich@hotmail.com,renanghp@gmail.com, liane@penta.ufrgs.br
}

How to cite this paper: Herpich, F., Guarese, R. L. M., \& Tarouco, L. M. R. (2017). A Comparative Analysis of Augmented Reality Frameworks Aimed at the Development of Educational Applications. Creative Education, 8, 1433-1451.

https://doi.org/10.4236/ce.2017.89101

Received: June 2, 2017

Accepted: July 24, 2017

Published: July 27, 2017

Copyright $\odot 2017$ by authors and Scientific Research Publishing Inc. This work is licensed under the Creative Commons Attribution International License (CC BY 4.0). http://creativecommons.org/licenses/by/4.0/

\begin{abstract}
This paper aims to analyze the existing frameworks that may allow the development of educational solutions using augmented reality resources, focusing on tools that enable the conception, design and implementation of mobile applications. As a result of this research, several development environments available on the market that facilitate working with augmented reality elements in mobile devices were discovered and examined. Among these platforms, eleven were selected to be analysed and presented in this paper. These were tested and compared to one another, and their main characteristics were indicated in a comparative table, as well as their resources that hold the potential to contribute with the construction of educational applications. All of this in favor of easing the development of applications that may assist educators in introducing augmented reality technologies in their classrooms.
\end{abstract}

\section{Keywords}

Augmented Reality (AR), Educational Applications, Software Development Kit (SDK), Mobile Devices, Edutainment, Virtual Reality (VR)

\section{Introduction}

The ever growing use of information and communication technologies on an educational scope has been modifying the traditional classroom scene, which brings up the need to think over the paradigms of computing in education. Following this perspective, several researches are being conducted with the intent of introducing new methods that may be used to improve the teaching and learning experiences. Among these is the intent of bringing augmented reality to pedagogical applications, providing the user a visualization, interaction and expe- 
rimentation of educational objects in scale, such as present by Nincarean et al. (2013), Wu et al. (2013), Zydney \& Warner (2016), and Jooste, Rautenbach \& Coetzee (2016).

Through augmented reality and its affordances, it's possible to amplify the channels of interaction between user and educational contents, enticing greater chances of learning. Some other benefits provided by augmented reality consist on reaching more elevated levels of user engagement, on the visualization of 3D virtual objects interposed to real ones, on the viewing of phenomena unperceivable in the real world in scale, as well as through different perspectives and angles. These characteristics assist the users in assimilating abstract and complex concepts, easing their comprehension of a given educational subject.

As augmented reality is being introduced in the educational scene and its pedagogical potential recognized, educators search new strategies to improve the user learning experience. With this in mind, Nincarean et al. (2013) add that augmented reality's efficacy may be improved when combined with other technologies, such as mobile devices, incorporating the typical features of these mobile technologies (e.g. portability, social interactivity, connectivity, context sensibility and individuality) with augmented reality's resources. This aspect, according to the authors, instigates the emergence of a new concept, called Mobile Augmented Reality (MAR).

Implementing educational applications with augmented reality resources on mobile devices brings up the need to choose a development framework capable of meeting the requirements and resources demanded by the project. Due to the great variety of tools available for the making of augmented reality software, it is legitimized the execution of a comparative analysis between them, in order to supply information to users about which augmented reality features each platform offers for the development of educational solutions in mobile devices.

In this manner, this paper has the objective of presenting a comparative analysis of eleven frameworks that offer the capability of developing augmented reality resources. It also has the intent of evidentiating the main features existing in this platforms and their potential for educational use, by showing their advantages and disadvantages in the development of educational applications on mobile devices.

\section{Augmented Reality}

Augmented Reality consists in the integration of virtual resources with real world physical elements, in which computer generated graphical components are presented in users' technological devices along with the real environment elements in loco. As established by Milgram \& Kishino (1994), as an operational definition of augmented reality, this may be the term considered to refer to any case in which an otherwise real environment is "augmented" by means of virtual objects (computer graphics).

As to achieve a more precise understanding of this area, it's possible to establish a contrast between the main characteristics of both virtual and augmented 
reality, an aspect which allows the demonstration of the fundamental difference between these two research areas. Virtual reality enables the user to feel the sensation of being immersed in a three-dimensional virtual environment, generated by and developed through a computer. Augmented reality, however, aims to combine the elements of a virtual environment with real world ones. Azuma (1997) claims that, in virtual reality, by being immersed in a synthetic environment, the user can't see the real world surrounding him. On the other hand, in augmented reality, the user can in fact see the real world, but with either virtual objects overlapping or composing it.

Such a dichotomy is presented and discussed by Milgram \& Kishino (1994) in Figure 1, where the authors demonstrate a trajectory named "Virtuality Continuum", referencing the mixture of object classes, representing the concept of a Mixed Reality. In this concept, real environments are in one end of the spectrum, whereas virtual ones are in the other far end. This shows the necessity of displaying real and virtual objects together in a unique exhibition as to visualize any environments localized in between the far ends of mixed reality.

By Milgram \& Kishino's (1994) definition, augmented reality may be interpreted as a midground between synthetic and real environments, in which AR complements virtual reality, instead of replacing it completely, giving the user the perception that virtual and real objects coexist in the same space. Referencing AR capable computational systems, Azuma (1997) argues that they should present three essential characteristics: a) combining virtual elements with the real environment; b) being interactive and having its process done in real time; and c) being conceived in three dimensions.

Regarding the uses and applications of augmented reality resources, it's possible to say that many studies are being conducted and that there are several computational solutions already assisting society, in many fields. Among these are areas like entertainment, marketing and advertising, tourism (Chung, Han \& Joun, 2015), automobilism (Rameau et al., 2016), health care (Jamali et al., 2015), training and education (Kysela \& Štorková, 2015), (Majid, Mohammed \& Sulaiman, 2015) and (Akçayir, Akçayir, Pektaş, \& Ocak, 2016), along with other areas (O'Shea \& Elliott, 2016), all of which are converging to the development of content and interactive solutions, allowing them to offer a pleasant and enriching experience to their users.

As reported, several are the applications that use AR resources, in many different areas, among which tend to stand out the ones focusing on an educational scope. Having in mind the focus of this paper, the next section will present some investigations that make use of these resources for the enhancement of the learning and teaching processes. It will also present a background of the area, as

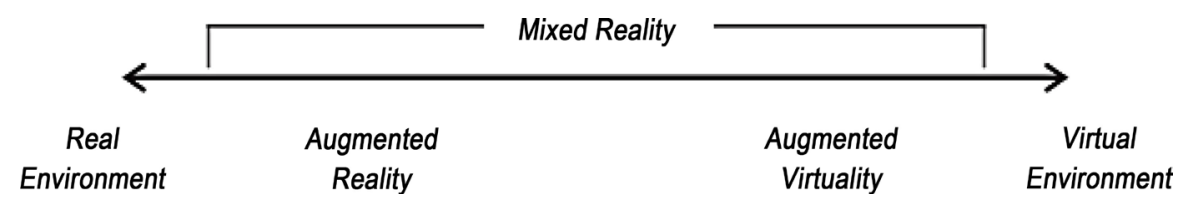

Figure 1. Virtuality Continuum (Milgram \& Kishino, 1994). 
well as the existing potentials that may be explored in favor of education.

\subsection{Augmented Reality in Education}

Many proposals that approach the using of augmented reality have been developed with the intention of improving the process of teaching and learning. In the same way, the combination of these resources with emerging technologies aimed at the educational area has been growing, such as mobile devices. For developing this research, some works were analysed in order to use the acquired knowledge for the formulation of the relevant criteria for the evaluation of the AR development frameworks.

The study presented by Kaufmann (2006) had the objective of addressing the integration of VR and AR technologies, combined with the intent of developing the Construct 3D environment, which aims to assist students in learning subjects related to geometry, more specifically topics such as dynamic three-dimensional geometry and spatial abilities. According to the author, the main advantage of employing AR resources consists in the fact that the student can now visualize the three-dimensional objects, which up to this point were calculated and drawn through traditional methods (such as pen and paper). The results achieved in three evaluations performed with professors and students showed that the environment is easy to be used, requires little time to be learned and encourages the students to properly explore geometry.

The researchers Irawati et al. (2008) present in their paper a 3D edutainment environment which provides an experience-based learning platform for understanding Newtonian physics laws. They designed a physics-based application that simulates the domino effect in a 3D environment. Using this application, the authors argue that users can learn physics by interacting and experiencing different kinds of domino effects in the VR/AR environment. There, it is presented an easy way for users to manipulate the simulation, showing the possible future trajectories of the selected objects. Based on these trajectories, the authors sustain the affirmation that it is possible to help the user to understand and compare the results of each selected configuration.

In Cai, Wang \& Chiang's (2014) research, the authors reported that, while teaching chemistry, students have difficulty in comprehending microworlds, as a consequence of their immature imaginative abilities, an aspect that influences in their understanding of microstructures and, therefore, makes this subject a great obstacle in their initial phase of learning chemistry. With the intent of easing these students' academic experience, the authors developed a set of learning tools based on AR, which enables them to control, combine and interact with a microparticles $3 \mathrm{D}$ model using augmented reality markers. While putting these tools to practice in a secondary school in China, the authors obtained positive results that their tools had a significant supplementary effect in the computer assisted learning and that it had a greater impact in low performance students than the other way around. Besides that, students showed a positive attitude regarding their learning experience using the software, which was positively correlated 
to their software evaluation.

This section presented some researches that are being developed with the support of augmented reality with an educational focus, demonstrating the benefits and advantages that this area is capable of providing to its users. Both in aspects related to the educational potential existing in these emerging technologies, as in the technical aspects, it was presented a know-how of the area, with the intention of offering the necessary fundamentation for the evaluation of the frameworks used in the development of these tools.

\subsection{Augmented Reality Frameworks}

Known as Software Development Kits (SDK), or simply frameworks, the tools used for the development of AR applications provide a coding environment where users are enabled to create the functionalities that will compose software applications with the capabilities and resources of augmented reality. According to Amin \& Govilkar (2015), these augmented reality SDKs facilitate many components within the AR application, for instance: AR recognition, AR tracking and $\mathrm{AR}$ content rendering.

In virtue of this area being in ascension and constantly present in current researches, there are several AR frameworks available in the market. There are, also, similar works that created comparisons looking to evidentiate the main existing AR platforms, such as Amin \& Govilkar (2015), Jooste, Rautenbach \& Coetzee (2016) and the collaborative construction of an online comparative table (Social Compare, 2017), However, all of these studies either presented a general outlook on AR or aimed their focus on tools that met their respective objectives. In short, all of them diverge from the objectives of this investigation, which means to present and analyse the frameworks whose potential may be applied to the construction of educational applications in mobile devices.

There are several tools currently in evidence for the execution of tasks that involve the development of applications that use AR resources. With this in mind, it was sought to present the main features demanded from tools that offer these resources, thus basing the platform selection that composes the comparative table and offering aids to the realization of comparative tests. In this next section, it will be present the methodological procedures adopted for the execution of tests and the further steps taken for the fulfillment of this research.

\section{Methodology}

The research design adopted for this investigation used the comparative method, with the intent of examining the main attributes and differences existing between the tools currently used for the development of educational applications with augmented reality resources.

\subsection{Analysed Tools}

A total of eleven frameworks, among AR viewers and browsers, were selected to compose the comparative analysis presented in this paper. The criterion adopted 
for selecting these frameworks was based in investigations conducted and in the analysis of the current AR scenery, aiming to highlight the frameworks with a better reputation in AR related researches and projects focused on the development of educational solutions.

For the framework selection, several evaluation criteria were established in order to observe which tools offered modern resources for implementing educational applications, constant updates, visibility in academic research and tools that allow the use of the features listed in the last section, being discarded all tools that were either terminated or discontinued, and the ones did not present an update in the last few years.

\subsection{Analysis and Tests Performed}

For a uniform analysis of the selected frameworks and their respective webbased management platforms, specific tests were conducted about each evaluated feature in the comparative chart, where it was verified whether or not the tool offers the established resource.

Furthermore, in virtue of this research having as one of its objectives to showcase platforms that facilitate the creation of AR applications within an educational scope, in order to execute the tests and assess the results, all tests referring to SDKs were done in the Unity 3D platform. Unity allows the user to export the source code to mobile device platforms, such as Android, iOS and Windows Phone, which enabled a more complete investigation of the AR frameworks.

During the execution of the tests, scores were assigned to the evaluation criteria considered relevant for developing educational AR applications. Because of this, nine requirements were considered for the evaluations, which are: text recognition, image recognition, 3D object recognition, multi-target recognition, geolocated tracking, markerless tracking, online target recognition, offline target recognition and AR editing platforms.

By the end of the assessment and with the intent of creating a graph comparing the frameworks presented in this paper, the established requirements were measured by their possible nomenclature answers for each evaluated criterion, consisting of "Yes" and "No". For all criteria that were assigned a "No", no points were attributed for the current platform being assessed. For the ones assigned a "Yes", however, 1 point was added to their score. The last evaluated parameter (AR Editing Platform), for being considered of greater relevance for the development of educational applications, had its number of points doubled for every framework that was assigned a "Yes" to it, thus receiving 2 points.

\section{Result Analysis}

For a better understanding of the evaluations performed and of the results obtained throughout this paper, the presentation and discussion of the outcome was divided into five stages. In the first phase, the AR development frameworks' functionalities chosen to be analysed were described and justified; in the second 
one, the frameworks selected to compose the comparative analysis were presented; in the third stage, it was presented the comparative table, the categories and the verified criteria; during the fourth one, the multimedia resources available in each framework were presented; and, finally, in the last phase, it was realized a broad discussion concerning the results, highlighting the frameworks that seemed the most prosperous in our comparison and showing their main attributes.

\subsection{Analysed Resources and Features}

Based on the investigations done by the authors of this paper in periodical publications and technical reports in the field, some characteristics considered relevant for the development of educational applications that use augmented reality resources were selected. Thus, the evaluation criteria present in Table 1 represent all of the aspects found in these authors' investigations that were considered relevant in a framework that means to offer developing tools for applications with AR features.

In the interest of composing a precise assessment, demonstrating the major attributes found in each framework and comparing them, it was established evaluation criteria in three distinctive categories, which were named "General information", "Marker identification and tracking" and "Additional Functionalities". In each of these classifications, subcategories were defined following a set of criteria with which it shall be produced a comparison. These are:

- Text Recognition: the frameworks were analysed on whether or not they offer the ability to recognize a word and/or a set of words.

- Image Recognition: in this criterion, the selected frameworks were submitted to a benchmark on their planar image recognition competences.

- 3D Object Recognition: the development platforms that allow the tracking of cylinder, conical and general real three-dimensional objects as markers were investigated.

- Multi-target Recognition: the frameworks that offer tracking of multiple targets simultaneously were analysed.

- Geolocation Recognition: tests were made in order to verify which tools support geolocated targets.

- Markerless Recognition: the selected development platforms were evaluated as to identify whether they support the use of any augmented reality technologies that do not require a marker.

- Online Target Recognition: tests were conducted in order to demonstrate which platforms make possible the tracking of targets available only through the internet in real time.

- Offline Target Recognition: the frameworks were investigated on the existence or not of resources that allow the recognition of targets in an offline manner, without any connection to the internet.

- AR Editing Platform: this subcategory intended to evaluate the selected frameworks that offer a ready to use web-based platform that allows the creation 
and management of markers, as well as the building and editing of augmented reality resources.

In the comparison shown in Table 1, another three criteria were mentioned. They were presented for a purely informative reason, with the intention of offering the user an additional knowledge about the frameworks, their versions, extensions and available platforms used throughout the tests, enabling even the possibility to reproduce the tests taken in this paper.

\subsection{Analysed Frameworks and Platforms}

For the sake of exposing the essential functionalities and applications of augmented reality in education, eleven tools were selected for analysis and tests. Among these, all allow the development of AR resources. In this manner, in order to compose a comparative table and create a basis for the discussion about the features available for the implementation of applications with an educational focus, the following are the frameworks that were analysed:

Table 1. A Comparative table of AR development tools.

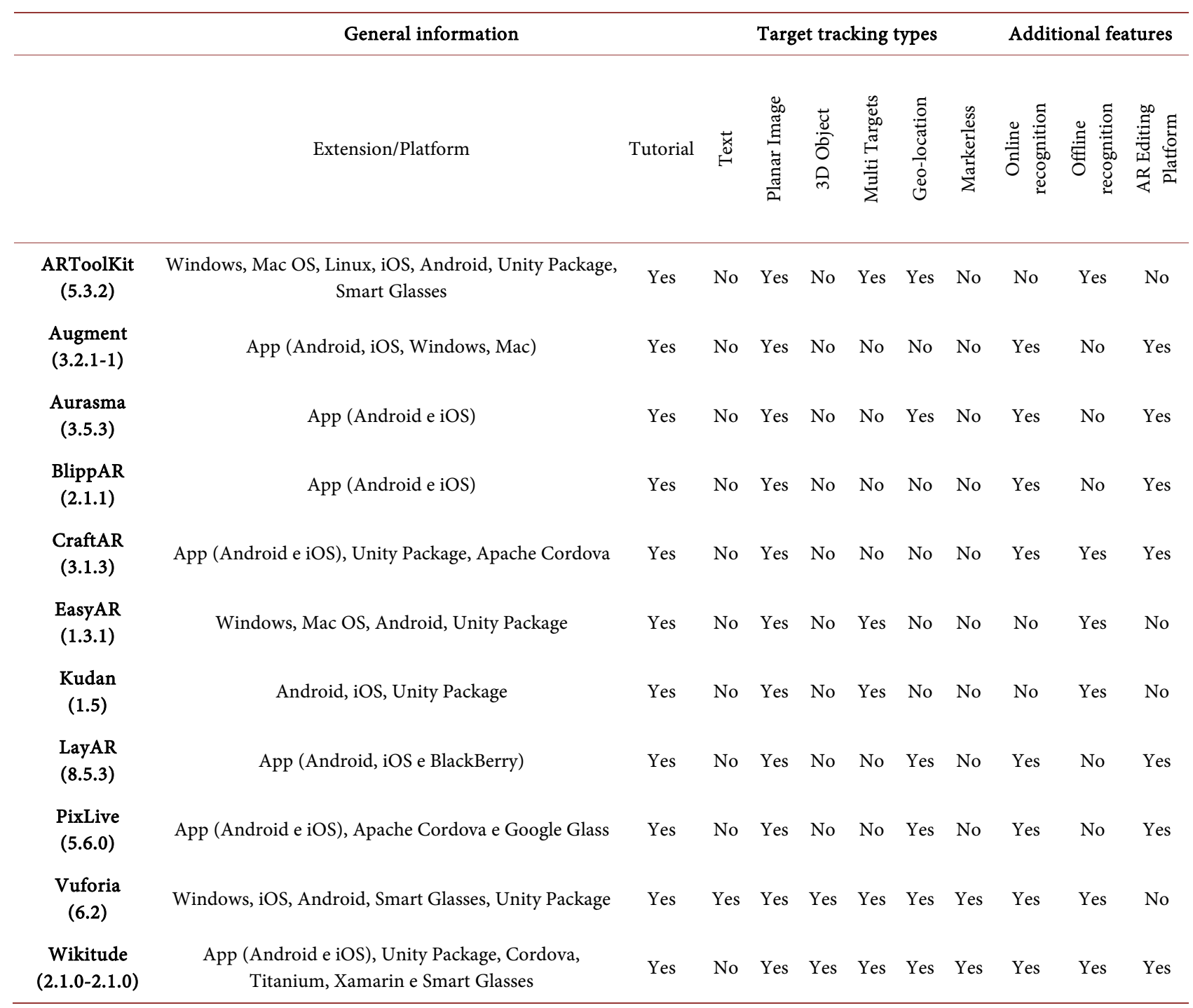


- ARToolKit: currently considered one of the most used frameworks for the development of applications with AR resources, in view of being an open source tool, being constantly updating, offering a series of features and allowing the exportation to other platforms. ARToolKit is capable of tracking planar, geolocated and multiple targets (ARToolKit, 2017).

- Augment: consists in an AR 3D viewer, composed by an application for mobile devices and a web-based platform, in which its users can register their own markers and associate them with 3D models and other virtual elements. Augment offers planar marker tracking and tracking via cloud storage (Augment, 2017).

- Aurasma: classified as an AR 3D viewer, Aurasma provides their own mobile application and a web-based platform, through which they allow their users to transform objects, images and places in new interactive opportunities using graphic content, animations, videos, audios and 3D content. It allows tracking of planar and geolocated targets, as well as tracking via cloud storage (Aurasma, 2017).

- BlippAR: it's an augmented reality 3D viewer that provides their own smartphone application and a web-based platform so that their users can register their markers and link them with many visual and interactive assets. BlippAR allows the user to track planar targets via cloud. It also instantiates a computer vision module with artificial intelligence and Deep Learning Algorithms, increasing the application's capacities by making possible that it eventually learns to recognize different things that the user may direct it towards (BlippAR, 2017).

- CraftAR: yet another AR 3D viewer, CraftAR offers their mobile app and a web-based platform so that users can easily manage and register their own markers, associating them with virtual content, e.g. 3D models, images, audio, video, etc. It also has an SDK supported by many platforms, making it possible that users develop their applications with CraftAR resources. It's capable of tracking planar images through the cloud (CraftAR, 2017).

- EasyAR: characterized as a development framework for AR based applications, EasyAR has a web-based platform through which users can register their projects and obtain the licenses needed to test and release their applications. It offers multiple planar target tracking (EasyAR, 2017).

- Kudan: it's categorized as a framework for developing applications with AR resources. Kudan offers an SDK that makes feasible the exporting to many other platforms, has a broad documentation and practical samples, besides having a support forum. It allows markerless tracking, using SLAM (Kudan, 2017).

- LayAR: consists in an augmented reality browser. It provides a mobile application and a web-based management platform, in which users can create and edit planar and geolocated targets and associate them to informative virtual contents such as text, URLs, audio and video (LayAR, 2017).

- PixLive: classified as an AR 3D viewer, PixLive offers a smartphone application 
and a web-based platform, through which users can create their own planar and/or geolocated targets. They can also associate them to digital resources, e.g. 3D models, images, audio, video, etc. (PixLive, 2017).

- Vuforia: also categorized as one of the most used frameworks for developing AR applications, besides having an SDK for several development platforms, Vuforia provides a web-based environment where users can create and manage their markers and obtain the licenses required to test and publish their applications. It's also capable of tracking planar, geolocated and multiple targets, texts and 3D objects, which can either be via cloud or locally stored in the user's device. Vuforia even allows markerless tracking, through two technologies: Extended Tracking and Smart Terrain (Vuforia, 2017).

- Wikitude: consists in an AR 3D viewer that provides the user with a mobile application and a web-based management platform, through which users are enabled to create their markers and link them to 3D models and other virtual elements. It also offers an SDK available in many development platforms, allowing the implementation of applications with Wikitude's resources. This framework is able to track planar markers, multiple targets, 3D objects, geolocated markers and it even makes use of markerless technology SLAM (Wikitude, 2017).

The tools described in this section correspond to a fraction of the total number of platforms analysed, once that other solutions (a total of 72 platforms) were verified and, in virtue of not attending the demanded criteria judged as relevant and required by the authors of this paper, were disregarded for the completion of the comparative scheme displayed in Table 1. In the next section, the aforementioned table will be presented and a discussion will be made based on the results observed in one of the evaluated categories.

\subsection{Comparative Analysis of Augmented Reality Tools}

The previously exposed functionalities approach the aspects that this paper's authors find relevant in a framework that means to offer the necessary resources for the development of applications that make use of augmented reality elements in an educational scope. In the interest of showing the disparity between the eleven analysed platforms presented in section 4.2 , it was built a comparative chart (Table 1), in which three general categories and eleven specific subcategories were established. This favored the investigation of the different aspects that may influence both the user's experience and the development of AR functionalities, which will be described in the following sections.

\subsubsection{General Information}

The "General Information" category proposes to expose the generic evaluation criteria under which the frameworks were analysed. These consist of the type of license in which each tool's resources are made available, which platforms and extensions are there and also the availability of support material and examples for the execution of tests.

In this manner, it's possible to observe that all platforms, with the exception of 
ARToolKit (open source) and EasyAR (free), make their functionalities available under proprietary licenses. However, it's important to discuss this aspect since, even being proprietary tools and charging for its services, all frameworks allow users to access and execute some tests, nonetheless some more advanced functionalities are either limited to premium accounts or available for a short trial period.

It was also observed which are the operational systems and platforms that each of the frameworks support and/or offer a plugin or extension for. Based on this, it was possible to evidentiate that all of them presented some solution for mobile devices, which is the focus of this paper, such as Android, BlackBerry and iOS. The portability of these tools for smart glasses platforms (such as Microsoft's Hololens) was also observed, whereas ARToolKit, PixLive, Vuforia and Wikitude were the tools that presented this possibility. Besides that, another interesting facet consists in the availability of Unity Packages, which allow the user to utilize the framework's components to develop his own applications in the widely used Unity platform, where he can export them to a wide range of devices and platforms.

Another important fact evinced by this category consists of the fact that all frameworks provide tutorials and examples to be followed for the implementation and use of its features, an attribute considered relevant by the authors of this paper, since this allows the users that are performing their first experiments or that don't have yet the experience to develop an application to obtain help and find the answers to their questions in either these tutorials or in the discussion forums of these platforms.

\subsubsection{Target Tracking Types}

This section is composed of one of the most relevant categories in our comparative analysis: the target tracking types. It should be mindfully observed during the selection of a framework for developing AR applications, once it attempts to present the tracking functionalities of each platform. Throughout the analysis, six different types of tracking were observed and inspected, all relevant to the building of educational applications, these were described in section 4.1 and will be discussing during the present section.

In this sense, it was possible to note that the subcategory related to textual tracking, i.e. the recognition of a word or a set of words, is solely by the Vuforia platform. This feature is emphasized as a resource useful for educational games for children, as well as a visual input mechanism (to be used in dictionaries and thesauruses, for instance), since Vuforia offers a list of over a hundred thousand words, commonly used in the english language, which can be incorporated to applications developed by the user (Vuforia, 2017).

Another aspect analysed during the conducted tests was the framework's capacity to track planar images as markers. This feature is present in all eleven of the frameworks that were analysed, which was already expected, since it is the most traditional way of tracking targets. It's also worth mentioning that platforms such as Vuforia, CraftAR and Wikitude allow the users to register their 
markers and then present a rating on the registered target, providing the user with a feedback on whether the rastreability elements of the image are adequate or not, so that they can be replaced by another one that attains a higher rating.

Regarding the tracking functionalities, another existing possibility is to track cylindrical, conical and other three dimensional targets. This tendency is presented with relevance, once it amplifies the tracking formats by including the possibility to recognize real objects in three dimensions and also enabling the use of perspective in augmented reality. Referencing this property, only two frameworks are capable of offering such feature: Vuforia and Wikitude. EasyAR mentions in its official page that it will include this resource in its next version (2.0).

A distinct important asset for AR developing tools is the opportunity to track multiple targets, a feature that allows the application in the user's device to maintain the tracking of more than one target at a time. As a result of this, besides being able to track a sequence of targets, the user becomes capable of also visualizing more than one virtual object in his device screen simultaneously, enabling even that one object complements or interacts with the other. Regarding this aspect, the platforms ARToolKit, EasyAR, Kudan, Vuforia and Wikitude all support multi-target tracking.

In virtue of the scope of this research presenting frameworks that allow the development and use of applications in mobile devices, the authors aimed to study and expose the platforms that are capable of exhibiting virtual elements based on the geolocation of the user's device. This functionality is often found in augmented reality games, e.g. Ingress (released on November 15, 2013) (2017) and Pokémon Go (released on July 6, 2016) (2017), in which the user needs to physically move to a determined position in order to be able to visualize the virtual elements associated to that geographical position, determined via the mobile device's GPS (Global Positioning System) signal. The platforms that allow the union of AR elements with geographical locations are ARToolKit, Aurasma, EasyAR, LayAR, PixLive, Vuforia and Wikitude.

Another feature analysed in the AR platforms was their capability of markerless tracking. This attribute becomes relevant in the moment that the user comprehends that it's not going to be necessary to point the device at a target, so merely pointing it to any free space where he wished to integrate the virtual element is enough. This enables the element to compose the scene in which it currently is, in order to enhance the user immersion in the experience proposed by the software, requiring less artificial elements, such as markers. Two platforms that provide this functionality are Kudan and Wikitude, both using algorithms to solve the SLAM (Simultaneous Localization and Mapping) problem. Another tool that offers this feature is Vuforia: by unifying its Extended Tracking and Smart Terrain technologies, it is also able to provide the user with markerless tracking. EasyAR mentions in its official webpage that it will support this sort of tracking in its next version (2.0).

The tools and their attributes discussed throughout this section contained the 
specificities related to the category "Target tracking types", in which it was meant to evaluate the essential tracking capabilities currently existent, from the most traditional methods of tracking, e.g. planar images and multi-targets, to the most advanced and contemporary ones, such as geolocated and markerless tracking. In this next section, the functionalities related to the management platforms of each one of the development tools for AR applications that are being analysed will be exposed.

\subsubsection{Additional Features}

The "Additional features" category presents information that the authors of this paper consider decisive for the moment during which the users make the choice of the AR development platform they are going to use. It discloses the resources that will help lay users in achieving positive final results using the augmented reality tools, e.g. the creation of targets and their association to virtual elements, such as images, audio, video and $3 \mathrm{D}$ models. In this sense, the AR platforms that offer the possibility of tracking targets were investigated and verified, being segmented into two categories: online and offline recognition. In virtue of both of these resources being considered important for the user's choice, through this division we manage to highlight the platforms that provide us with both tracking services.

In relation to online target recognition, the platforms that offer this feature are as follows: Augment, Aurasma, BlippAR, CraftAR, LayAR, PixLive, Vuforia and Wikitude. The remaining tools, i.e. ARToolKit, EasyAR and Kudan, allow only offline target tracking, which requires the developer to insert the targets in the application via code, storing the target images in the user's device. Besides the aforementioned platforms, Augment, CraftAR, Vuforia and Wikitude are also capable of offline tracking, which makes the latter four platforms the only ones to allow both online and offline tracking, an aspect that greatly amplifies the adaptability of this framework to different user needs. EasyAR announces in its official webpage that it will support both methods of tracking in its next version (2.0).

Tracking targets without the need to connect to the internet has its benefits and detriments. On the one hand, it becomes a great advantage to have the markers stored directly in the device, since it not only makes tracking faster, but it also allows the user to track targets and visualize the virtual elements even when his internet connection fails or simply isn't there, without compromising his augmented reality experience. On the other hand, introducing markers directly into the source code not only complicates the development of the application, but it also increases its dimension, i.e., the bigger the number of markers attached to the application, the more data it will require to be downloaded and stored in the user's device. The recognition of targets stored online also presents an important question to be considered, which is based on the device's necessity to be connected to the internet in order to be able to both track any marker and load the AR virtual elements associated with it.

The last criterion listed to the category of additional features consists of 
analysing the frameworks that enable the creation and edition of markers and augmented reality resources in their web-based platforms. By this, we mean to indicate which tools allow the user to register and manage his markers in a way that, once registered, the markers can be used in the his projects as targets. In accordance to this criterion, the frameworks that let the user to edit and produce AR elements right in the same platform were also observed, thus allowing the developer to complete the whole process in a unique standalone platform.

The inclusion of this last criterion is justified by the fact that this paper also aims to expose frameworks that may assist a user that lacks the experience of developing applications, especially educators that wish to present innovative contents in the classroom. Through this sort of resource, it becomes possible to speed up both the development of the application and the production of courseware. Among the analysed AR platforms, seven offer this sort of functionality: Augment, Aurasma, BlippAR, CraftAR, LayAR, PixLive and Wikitude. Between the remaining ones, ARToolKit, EasyAR and Kudan also use markers, but they do not facilitate their creation in their platform. The markers can only be stored directly in the software's source code, which hinders the creation process done by new developers and people who lack the knowledge of system development. Vuforia also doesn't fully meet this requirement, nevertheless, it does permit markers to be both declared in the source code as well as registered in their website, either to be downloaded as a package ready to be used in the developer's project or to be tracked as a target via cloud.

Still on the subject, EasyAR announces in its official page that it will provide this resource in their next version (2.0), while ARToolKit supplies a training software to teach the user about target tracking algorithms. This software, however, is neither automated nor practical, since it requires the user to fully follow the training and then manually insert the coding results in his application.

The "Additional features" category discussed throughout this section had the intention of displaying relevant information to assist in the selection of an AR development framework that should satisfy all of the user's aspirations and serve him in the best way possible as to build educational applications.

Regarding the tools that presented the "AR editing platform" feature, an analysis was made about the multimedia resources and other virtual elements that each of the seven platforms offer to their users. This will be better approached in the next section.

\subsection{Multimedia Resources}

Based on the attributes demonstrated in the last section, specifically in Table 1, it was indispensable to present a discussion regarding the augmented reality frameworks which enable the user create and edit 3D models and other AR elements directly in their web-based platform. Therefore, this section aims to expose the multimedia resources offered by each of the augmented reality frameworks that supply an AR editing platform, demonstrating the virtual elements available and their supported formats, as well as any other characteristics that 
may be relevant for developing educational applications. The main properties found in each framework were listed in Table 2.

Ascertained by the knowledge displayed in Table 2, the information demonstrated in the comparative chart and the analysis of the available resources, it's possible to offer a broad know-how to the final user, enabling him to choose the set of tools that best attends to his project requirements and resource needs. All of this aiming to accomplish our intent of assisting the development of educational applications using augmented reality.

\subsection{Result Discussion}

Based on the comparative analysis of both the features and the virtual elements available for the development of mobile applications with AR assets, it's possible

to establish some general observations concerning the tools analysed in this

Table 2. Virtual resources description of the web-based platforms.

\begin{tabular}{|c|c|}
\hline Framework & Media \\
\hline Augment & $\begin{array}{l}\text { Allows the association of the marker to a } 3 \mathrm{D} \text { mode, URL or folder. It } \\
\text { offers limited options of settings for the } 3 \mathrm{D} \text { models, impeding great } \\
\text { modifications. }\end{array}$ \\
\hline Aurasma & $\begin{array}{l}\text { Allows the association of touching actions on the screen, to start and end } \\
\text { the app and after a determined amount of time. } \\
\text { For example, when an element that composes the scene is touched by the } \\
\text { user, it's possible to open a webpage in the device's browser, just like } \\
\text { other basic functionalities. }\end{array}$ \\
\hline BlippAR & $\begin{array}{l}\text { Allows the addition of buttons for social media, web pages, telephone } \\
\text { calls and shopping. } \\
\text { Enables the use of media such as images, audio, video and supports } \\
\text { Youtube, Sound Cloud, Spotify and other media platforms. } \\
\text { Allows the creation of scenes and buttons to guide the user between } \\
\text { them. All of the resources used can be configured for a better } \\
\text { presentation. It also supports layer animations. }\end{array}$ \\
\hline
\end{tabular}

CraftAR Allows adding buttons, images, videos and 3D models. In the premium version, CraftAR enables statistics about the markers and user access.

LayAR Allows the addition of buttons for social media, web pages, telephone calls, votings, shopping, emails, downloads and adding contacts. Enables the use of media such as images, audio and video. In the premium account, LayAR lets you add elements in HTML, run applications and use geo-located resources.

PixLive Allows adding buttons for social media, web pages, PDF files and using text and images as buttons.

Enables the creation of scenes and buttons or timers to navigate between the scenes. Allows the use of media such as images, $360^{\circ}$ images, $3 \mathrm{D}$ models, audio and video. Allows the use of geo-located resources and drawing applications.

Wikitude Enables the use of media such as images, video and 3D models. Allows the insertion and edition of text and buttons for social media.

3D models format accepted: Collada (.dae and.zae), Wavefront OBJ (.obj), Stereolitography (.stl) and.KMZ.

Images file format accepted: JPG,. JPEG, BMP, PNG, GIF

3D models format accepted: Collada (.dae). Images file format accepted: PNG.

Doesn't specify the supported video formats

Images file format accepted: Filmbox (.fbx).

3D models format accepted: Wavefront OBJ (.obj) no formato Zip.

Videos file format accepted: H.264/MP4 and Youtube.

Images file format accepted: JPG and PNG.

Audios file format accepted: MP3 and

SoundCloud.

3D models format accepted: Collada (.dae), 3DS Max (.3ds), Wavefront OBJ (.obj),

Stereolitography (.stl).

Videos file format accepted: H.264/MP4, Youtube,

Vimeo and Dailymotion.

Doesn't specify the supported formats. 


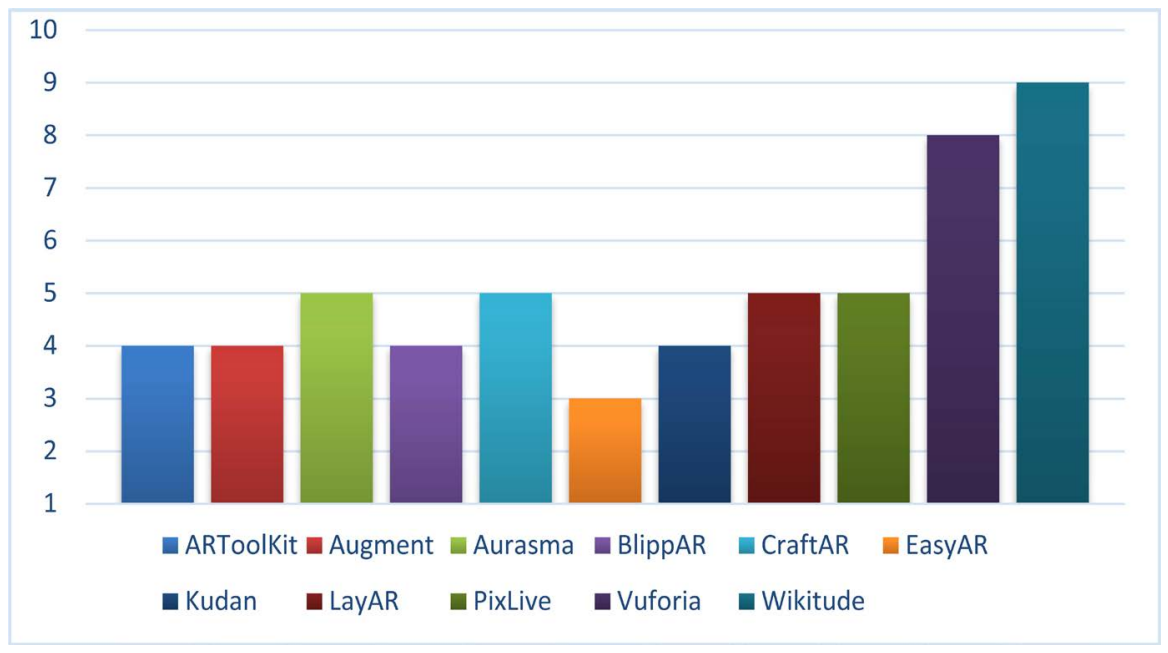

Graph 1. A Comparative chart of AR development tools.

study. In order to demonstrate the results in a more meaningful manner, a graphical representation (Graph 1) was made, which presents all AR platforms analysed in this paper, exposing a quantitative score for each tool representing the proportions reached by them in the criteria that they were analysed.

Taking into account the values obtained in the comparative chart and displayed in Graph 1, it can verified that some augmented reality frameworks that are widespread in researches and, in a general sense, in academia, scored lower grades when compared to other platforms, remaining in an inferior rating zone. This is the case of ArtoolKit (which scored 4 points), EasyAR (score of 3) and Kudan (score of 4). Ascertained by the ex-posed evidences, these scores can be justified by the fact that all three frameworks are intended for developers with experience in system development (since they are SDKs). This aspect makes them more complex in relation to the other analysed frameworks, re-sulting in inferior scores, once that this investigation pursues to highlight frameworks that can be used by users that not necessarily have developed software before. This allows an easier and faster creation of educational applications.

Another two frameworks that were classificated at the lower scoring zone were Augment and BlippAR, both with a score of four. Regarding these two frameworks, their low ratings can be explained by the fact that both of them present only one option for tracking targets: planar images, an aspect that badly influenced their final ranking. On the other hand, Augment and BlippAR both enable the user to track targets online and to create and edit AR elements in their web-based platforms, which can indeed positively influence the user's choice.

Concerning the augmented reality platforms that remained in the intermediate ranking zone, which were Aurasma, CraftAR, LayAR and PixLive (all with a rating of 5), it was possible to perceive in Table 1 that all of them are able to track planar images, via cloud, being CraftAR also able to track targets offline. Aurasma, LayAR and PixLive also allow the creation and use of geo-located markers, using the device's GPS to recognize targets. Furthermore, all four platforms 
offer the user a web-based platform to create and edit their AR elements.

In regard of the top rated frameworks, Vuforia (8 points) and Wikitude ( 9 points), based on Table 1, it's possible to notice that their resources referring to the target tracking types category caused their distinction among the other platforms, in virtue of both of them allowing the recognition of planar images, three-dimensional objects, geo-located markers, multi-target and markerless tracking, and both online and offline target tracking. The only disparity between Vuforia and Wikitude is that the former is able to recognize and interpret textual targets, while the latter offers a web-based platform for creating and editing AR elements, a characteristic which made it better qualified in our quantitative ranking.

The discussion presented during this section intended to better contrast the existing differences between the AR frameworks analysed in this paper, exposing their main attributes and presenting their advantages and disadvantages, aiming to highlight the existent potential in each tool for the development of applications in an educational scope. Besides that, through the comparison presented in Graph 1, the authors of this paper aspire to offer the necessary information so that users can choose the platform that best suits their requirements for augmented reality resources and functionalities.

\section{Conclusion}

With the constant evolution of information technology and communication in the most varied scopes of application, new paradigms are being created to assist the processes of teaching and learning. Augmented reality is also converging this way, looking forward to contribute for the evolution of educational technologies, as well as of the courseware available to students, through virtual resources and a bigger interactivity, besides its great propagation made possible when adopted by mobile technologies.

This work summarizes a comparative analysis of frameworks that can be used to develop applications with $\mathrm{AR}$ resources, presenting as a result a total of eleven tested and compared platforms, which were selected with a perspective focused on their capacity to develop applications for mobile devices. Their main attributes were exposed, as well as the virtual and augmented resources that may assist in the implementation of modern, engaging and immersive educational applications. As a result of this analysis, it was possible to perceive that the platforms Wikitude and Vuforia met the greatest number of selected requirements, making available a vast array of multimedia resources for the building of AR applications focused on education.

During the tool analysis, another discovery found relevant for the current theme of this research is related to the eventual difficulty faced by professionals that lack the experience in the area of computational solutions while trying to build software for educational purposes. These can include all professionals such as educators and researchers of any area remote to computation. This aspect arises constantly as a distress for these people that aim to build innovative solutions for 
their classrooms. However, based on the gathering of information presented in this paper, it's possible to claim that the AR developing tools that offer an AR Editing Platform may ease or even make possible the high-level development process of educational softwares, i.e., it might facilitate the operation of creating software without requiring the knowledge needed to implement algorithms. This becomes true once that these platforms do not require the creator to write any code for the algorithms, demanding only that he adds AR resources, such as virtual media and three-dimensional models, to a web-based management platform. Among these tools are Augment, Aurasma, BlippAR, CraftAR, LayAR, PixLive and Wikitude.

Regarding to future perspectives, this paper intends to continue its investigations about AR frameworks and functionalities, exposing new technologies and their possible use for building applications in an educational scope. Another investigation that will be conducted in a future research consists of the runtime each of these frameworks take during the analysis and recognition of targets.

\section{Acknowledgements}

This research is supported by CNPq (National Council for Scientific and Technological Development), a Brazilian government entity focused on scientific development.

\section{References}

Akçayir, M., Akçayir, G., Pektaş, H. M., \& Ocak, M. A. (2016). Augmented Reality in Science Laboratories: The Effects of Augmented Reality on University Students' Laboratory Skills and Attitudes toward Science Laboratories. Computers in Human Behavior, 57, 334-342. https://doi.org/10.1016/j.chb.2015.12.054

Amin, D., \& Govilkar, S. (2015). Comparative Study of Augmented Reality SDK's. International Journal on Computational Sciences \& Applications, 5, 11-26.

https://doi.org/10.5121/ijcsa.2015.5102

ARToolKit (2017). Official ARToolKit Web Page. https://artoolkit.org/

Augment (2017). Official Augment Web Page. http://www.augment.com/

Aurasma (2017). Official Aurasma Web Page. https://www.aurasma.com/

Azuma, R. (1997). A Survey of Augmented Reality. Presence: Teleoperators and Virtual Environments, 6, 355-385. https://doi.org/10.1162/pres.1997.6.4.355

BlippAR (2017). Official BlippAR Web Page. https://blippar.com/en/

Cai, S., Wang, X., \& Chiang, F. K. (2014). A case Study of Augmented Reality Simulation System Application in a Chemistry Course. Computers in Human Behavior, 37, 31-40. https://doi.org/10.1016/j.chb.2014.04.018

Chung, N., Han, H., \& Joun, Y. (2015). Tourists' Intention to Visit a Destination: The Role of Augmented Reality (AR) Application for a Heritage Site. Computers in Human Behavior, 50, 588-599. https://doi.org/10.1016/j.chb.2015.02.068

CraftAR (2017). Official CraftAR Web Page. https://catchoom.com/product/craftar/augmented-reality-and-image-recognition/

EasyAR (2017). Official EasyAR Web Page. http://www.easyar.com/

Ingress (2017). Official Ingress Web Page. https://www.ingress.com/ 
Irawati, S., Hong, S., Kim, J., \& Ko, H. (2008). 3D Edutainment Environment: Learning Physics through VR/AR Experiences. In Association for Computing Machinery (Ed.), Proceedings of the 2008 International Conference on Advances in Computer Entertainment Technology (pp. 21-24). New York: Association for Computing Machinery. https://doi.org/10.1145/1501750.1501755

Jamali, S. S., Shiratuddin, M. F., Wong, K. W., \& Oskam, C. L. (2015). Utilising MobileAugmented Reality for Learning Human Anatomy. Procedia-Social and Behavioral Sciences, 197, 659-668. https://doi.org/10.1016/j.sbspro.2015.07.054

Jooste, D., Rautenbach, V., \& Coetzee, S. (2016). Results of an Evaluation of Augmented Reality Mobile Development Frameworks for Addresses in Augmented Reality. Spatial Information Research, 24, 1-13. https://doi.org/10.1007/s41324-016-0022-1

Kaufmann, H. (2006). The Potential of Augmented Reality in Dynamic Geometry Education. The 12th International Conference on Geometry and Graphics (ISGG) (pp. 1-14). Salvador, Brazil.

Kudan (2017). Official Kudan Web Page. https://www.kudan.eu/

Kysela, J., \& Štorková, P. (2015). Science Direct Using Augmented Reality as a Medium for Teaching History and Tourism. Procedia-Social and Behavioral Sciences, 174, 926931. https://doi.org/10.1016/j.sbspro.2015.01.713

LayAR (2017). Official LayAR Web Page. https://www.layar.com/

Majid, N. A. A., Mohammed, H., \& Sulaiman, R. (2015). Students' Perception of Mobile Augmented Reality Applications in Learning Computer Organization. Procedia-Social and Behavioral Sciences, 176, 111-116. https://doi.org/10.1016/j.sbspro.2015.01.450

Milgram, P., \& Kishino, F. (1994). Taxonomy of Mixed Reality Visual Displays. IEICE Transactions on Information and Systems, E77, 1321-1329. https://doi.org/10.1.1.102.4646

Nincarean, D., Ali, M. B., Dayana, N., Halim, A., Hishamuddin, M., \& Rahman, A. (2013). Mobile Augmented Reality: The Potential for Education. Procedia-Social and Behavioral Sciences, 103, 657-664. https://doi.org/10.1016/j.sbspro.2013.10.385

O'Shea, P. M., \& Elliott, J. B. (2016). Augmented Reality in Education: An Exploration and Analysis of Currently Available Educational Apps. In C. Allison, L. Morgado, J. Pirker, D. Beck, J. Richter, \& C. Güetl (Eds.), Communications in Computer and Information Science (Immersive Learning Research Network: Second International Conference (iLRN)) (2nd ed., pp. 147-159). Santa Barbara, CA: Springer International Publishing. https://doi.org/10.1007/978-3-319-41769-1_12

PixLive (2017). Official PixLive Web Page. https://www.vidinoti.com/

PokémonGo (2017). Official PokémonGo Web Page. http://www.pokemongo.com/

Rameau, F., Ha, H., Joo, K., Choi, J., Park, K., \& Kweon, I. S. (2016). A Real-Time Augmented Reality System to See-Through Cars. IEEE Transactions on Visualization and Computer Graphics, 22, 2395-2404. https://doi.org/10.1109/TVCG.2016.2593768

SocialCompare (2017). Official SocialCompare Web Page. http://socialcompare.com/en/comparison/augmented-reality-sdks/

Vuforia (2017). Official Vuforia Web Page. https://www.vuforia.com/

Wikitude (2017). Official Wikitude Web Page. https://www.wikitude.com/

Wu, H. K., Lee, S. W. Y., Chang, H. Y., \& Liang, J. C. (2013). Current Status, Opportunities and Challenges of Augmented Reality in Education. Computers and Education, 62, 41-49. https://doi.org/10.1016/j.compedu.2012.10.024

Zydney, J. M., \& Warner, Z. (2016). Mobile Apps for Science Learning: Review of Research. Computers \& Education, 94, 1-17. https://doi.org/10.1016/j.compedu.2015.11.001 
Submit or recommend next manuscript to SCIRP and we will provide best service for you:

Accepting pre-submission inquiries through Email, Facebook, LinkedIn, Twitter, etc. A wide selection of journals (inclusive of 9 subjects, more than 200 journals)

Providing 24-hour high-quality service

User-friendly online submission system

Fair and swift peer-review system

Efficient typesetting and proofreading procedure

Display of the result of downloads and visits, as well as the number of cited articles Maximum dissemination of your research work

Submit your manuscript at: http://papersubmission.scirp.org/

Or contact ce@scirp.org 